\title{
VARICOSE VEINS OF LOWER EXTREMITIES IN PREGNANT WOMEN AND BIRTH OUTCOMES
}

\author{
Ferenc Bánhidy, Nándor Ács, Erzsébet H. Puhó, Andrew E. Czeizel \\ Second Department of Obstetrics and Gynecology, Semmelweis University, School of Medicine, Budapest, Hungary, and Foundation for the \\ Community Control of Hereditary Diseases, Budapest, Hungary
}

\begin{abstract}
SUMMARY
The objective of the study was to estimate the association of pregnant women with varicose veins of lower extremities (VVLE) and the possible risk for adverse birth outcomes and among them different congenital abnormalities (CAs) in their children. Prospectively and medically recorded VVLE were evaluated in 332 pregnant women who delivered infants with CA (case group) and 566 pregnant women with VVLE who delivered infants without CA (control group) and matched to cases were compared in the population-based data set of the Hungarian Case-Control Surveillance System of Congenital Abnormalities, 1980-1996. About one-quarter of pregnant women had chronic VVLE while new onset VVLE occurred in the rest of pregnant women. There was no higher risk for adverse birth outcomes of pregnant women with VVLE, in fact the rate of preterm birth and low birth weight was somewhat lower than in the newborns of pregnant women without VVLE. The comparison of VVLE occurrence in pregnant women who had offspring with 21 different CA groups and in pregnant women who later delivered babies without CA showed a higher risk only for pectus excavatum, a mild CA. In conclusion, VVLE in pregnant women does not associate with obvious hazard for their fetuses.
\end{abstract}

Key words: varicose veins of lower extremities, related drug treatment, bith outcomes, congenital abnormalities, pectus excavatum

Address for correspondence: A. E. Czeizel, Törökvész lejtő 32, H-1026 Budapest, Hungary. E-mail: czeizel@ interware.hu

\section{INTRODUCTION}

Primary varicosities, i.e. dilated veins with valve insufficiency tend to be familial and frequently occur without other causative events (1). Varicose veins may occur at several sites of the body, e.g. in the scrotum, vulva, oesophagus, but most frequently in the lower extremities. The definition of varicose veins of lower extremities (VVLE) is abnormal dilatation and formation vena saphena magna, and rarely vena saphena parva in the lower limbs with the symptoms of burning, bursting, bruised or aching of patients (1).

VVLE is about twice as frequent in females as in males. In addition pregnancy induces dilatation and proliferation of blood vessels, therefore venous congestion and increased vascular permeability during pregnancy commonly cause edema of the skin and subcutaneous tissue, particularly vulva and lower legs (2). VVLE often first appears during pregnancy.

VVLE were recorded frequently in Hungarian pregnant women, nevertheless, we were not able to find the results of controlled epidemiological studies regarding the effect of VVLE in pregnant women for birth outcomes (3). Thus the objective of our study was to evaluate the possible association between maternal VVLE recorded in the population-based data set of the Hungarian CaseControl Surveillance of Congenital Abnormalities (HCCSCA) (4) and adverse birth outcomes, particularly the risk of structural birth defects, i.e. congenital abnormalities (CAs) in their children.

\section{MATERIALS AND METHODS}

Cases affected with CA were selected from the data set of the Hungarian Congenital Abnormality Registry (HCAR), 1980-1996
(5) for the HCCSCA. Notification of cases with CA is mandatory for physicians from the birth until the end of first postnatal year to the HCAR. Most cases are reported by obstetricians and paediatricians. In Hungary practically all deliveries take place in inpatient obstetric clinics and the birth attendants are obstetricians. Paediatricians are working in the neonatal units of inpatient obstetric clinics, or in various inpatient and outpatient paediatric clinics. Autopsy was mandatory for all infant deaths and common in stillborn fetuses during the study period. Pathologists sent a copy of the autopsy report to the HCAR if defects were identified in stillbirths and infant deaths. Since 1984 fetal defects diagnosed in prenatal diagnostic centres with or without termination of pregnancy have also been included into the HCAR.

CAs were split up into three groups: lethal (if defects cause stillbirths or infant deaths or pregnancies were terminated due to fetal defect in more than $50 \%$ of cases), severe (without medical intervention CAs cause handicap or death) and mild (CAs require medical intervention but life expectancy is good). Lethal and severe CAs together constitute major CAs. (Minor anomalies or morphological variants without serious medical or cosmetic consequences are excluded from the class of CA.) In addition two main categories of cases with CAs were differentiated: isolated (only one organ is affected) and multiple (concurrence of two or more CAs in the same person affecting at least two different organ systems).

The total (birth + fetal) prevalence of cases with CA diagnosed from the second trimester of pregnancy through the age of one year was 35 per 1,000 informative offspring (live-born infants, stillborn fetuses and electively terminated malformed fetuses) in the HCAR, 1980-1996 (5), though the expected rate was 65.3 per 1,000 (6). However, about $90 \%$ of major CAs were believed to be recorded in the HCAR during the 17 years of the study period (5). 
There were three exclusion criteria of cases with CAs from the HCAR for the data set of the HCCSCA. (i) Cases reported after three months of birth or pregnancy termination (the longer time between birth or pregnancy termination and data collection decreases the accuracy of information about pregnancy history and $77 \%$ of cases were reported during the first three-month time window, the rest included mainly mild CAs). (ii) Cases with three mild CAs (congenital dysplasia of hip and inguinal hernia, large haemangioma), and (iii) CA-syndromes caused by major mutant genes or chromosomal aberrations with preconceptional origin.

Controls were defined as newborn infants without CA and they were selected from the National Birth Registry of the Central Statistical Office for the HCCSCA. In general two controls were matched to every case according to sex, birth week, and district of parents' residence.

Three sources of exposure and confounding data were evaluated:

\section{Prospective Medically Recorded Data}

Mothers were asked in an explanatory letter to send us the prenatal maternity logbook and other medical records particularly discharge summaries concerning their diseases during the study pregnancy and their child's CA. Prenatal care is mandatory for pregnant women in Hungary (if pregnant women did not visit prenatal care clinic, she did not receive a maternity grant and leave), thus nearly $100 \%$ of pregnant women visited prenatal care clinics, on average 7 times in their pregnancies. The first visit was between the 6th and 12th gestational week. The task of obstetricians is to record all pregnancy complications, maternal diseases and related drug prescriptions in the prenatal maternity logbook.

\section{Retrospective Self-Reported Maternal Information}

A structured questionnaire with a list of medicinal products (drugs and pregnancy supplements) and diseases, plus a printed informed consent form were also mailed to the mothers immediately after the selection of cases and controls. The questionnaire requested information on pregnancy complications and maternal diseases, on medicinal products taken during pregnancy according to gestational months, and on family history of CAs. To standardize the answers, mothers were asked to read the enclosed lists of medicinal products and diseases as a memory aid before they filled out the questionnaire.

The mean + S.D. time elapsed between the birth or pregnancy termination and the return of the "information package" (logbook, questionnaire, discharge summary, and informed consent form) in our prepaid envelope was 3.5+1.2 and 5.2+2.9 months in the case and control groups, respectively.

\section{Supplementary Data Collection}

Regional nurses were asked to visit all non-respondent case mothers and to help mothers to fill out the same questionnaire used in the HCCSCA, to evaluate the available medical records, and to obtain data regarding smoking and drinking habit through a cross interview of mothers and their close relatives living together, and the so-called 'family consensus' was evaluated. Regional nurses visited only 200 non-respondent and 600 respondent control moth- ers as part of two validation studies $(7,8)$, because the committee on ethics considered this follow-up to be disturbing to the parents of all healthy children. Regional nurses used the same method at the visit of control mothers and case mothers.

Overall, the necessary information was available on $96.3 \%$ of cases (84.4\% from reply to the mailing, $11.9 \%$ from the nurse visit) and $83.0 \%$ of the controls (80.9\% from reply, $2.1 \%$ from visit). Informed consent form was signed by $98 \%$ of mothers, names and addresses were deleted in the rest $2 \%$.

Here the 17 years' data of the HCCSCA between 1980 and 1996 are evaluated because the data collection has been changed since 1997 (all mothers are visited by regional nurses), and the recent data had not been validated at the time of analysis.

The Hungarian medical doctors use the international diagnostic criteria of VVLE. The CEAP (clinical manifestation, etiologic factors, anatomic involvement and pathophysiological feature) classification scheme for lower-extremity venous diseases differentiates six groups $(9,10)$ : C1: teleangiectasia or reticular veins, C2: varicose veins, C3: edema without skin changes, C4a: skin pigmentation or eczema, C4b: lipodermatosclerosis or atrophie blanche, C5: healed ulceration, and C6: active ulceration.

The objective of our study was the evaluation of VVLE, which corresponded to $\mathrm{C} 2$. At the preliminary analysis of our data we excluded pregnant women who had varicose veins on other sites (e. g. oesophageal and vulvar varices), teleangiectasias, spider angioma, secondary varicosities due to extrinsic venous compression, prior deep vein thrombosis, congenital vascular lesions, etc. and VVLE associated with phlebitis and thrombophlebitis (11).

In addition two groups of VVLE could be differentiated according to the source of information in the HCCSCA: (i) prospectively and medically recorded VVLE in the prenatal maternity logbook and (ii) VVLE based on only retrospective maternal information. However, the spectrum of VVLE is very wide, in addition the perception of VVLE depends on the sensitivity of pregnant women, therefore the validity of VVLE diagnosis was low in the latter group (7). Thus we decided to evaluate only prospectively and medically recorded VVLE in the prenatal maternity logbook, obviously this diagnosis covered the most severe VVLE, rarely healed ulceration. However, VVLE with inflammation, or active ulceration was not recorded in the prenatal maternity logbook of pregnant women in the data set of the HCCSCA.

VVLE related drug treatments were also evaluated. Other potential confounding factors included maternal age, birth order, marital and employment status as indicators of socio-economic status because they showed a good correlation with the level of education and income (12), maternal diseases, pregnancy supplements particularly folic acid and multivitamins were considered as indicators of the standard of preconceptional and prenatal care, in addition as a primary preventive method of some CAs after their periconceptional use. Finally the possible association of VVLE with medically recorded pregnancy complications was also evaluated.

Gestational age was calculated from the first day of the last menstrual period. The critical period of most major CAs is in the second and/or third months, it explains that this time window as the critical period of CAs was used in the study $(13,14)$. Beyond birth weight (g) and gestational age at delivery (wk), the rate of low birthweight newborns ( $<2500 \mathrm{~g})$ and preterm births $(<37$ weeks) were analyzed on the basis of discharge summaries of inpatient obstetric clinics. 
Table 1. Maternal characteristics of case and control mothers with VVLE during the study pregnancy

\begin{tabular}{|c|c|c|c|c|c|c|c|c|}
\hline \multirow[t]{2}{*}{ Variables } & \multicolumn{4}{|c|}{ Case mothers } & \multicolumn{4}{|c|}{ Control mothers } \\
\hline & \multicolumn{2}{|c|}{ without } & \multicolumn{2}{|c|}{ with } & \multicolumn{2}{|c|}{ without } & \multicolumn{2}{|c|}{ with } \\
\hline & \multicolumn{4}{|c|}{ VLE } & \multicolumn{4}{|c|}{ VVLE } \\
\hline Quantitative & \multicolumn{2}{|c|}{$(\mathrm{N}=22,511)$} & \multicolumn{2}{|c|}{$(\mathrm{N}=332)$} & \multicolumn{2}{|c|}{$(\mathrm{N}=37,585)$} & \multicolumn{2}{|c|}{$(\mathrm{N}=566)$} \\
\hline Maternal age, yr. & No. & $\%$ & No. & $\%$ & No. & $\%$ & No. & $\%$ \\
\hline-19 & 2,496 & 11.1 & 10 & 3.0 & 3,258 & 8.7 & 19 & 3.4 \\
\hline $20-29$ & 15,403 & 68.4 & 190 & 57.2 & 27,264 & 72.5 & 338 & 59.7 \\
\hline $30-$ & 4,612 & 20.5 & 132 & 39.8 & 7,063 & 18.8 & 206 & 36.9 \\
\hline Mean \pm S.D. & \multicolumn{2}{|c|}{$25.4+5.3$} & \multicolumn{2}{|c|}{$28.5+5.3$} & \multicolumn{2}{|c|}{$25.4+4.9$} & \multicolumn{2}{|c|}{$28.0+5.2$} \\
\hline \multicolumn{9}{|l|}{ Birth order (parity) } \\
\hline 1 & 10,639 & 47.3 & 69 & 20.8 & 18,090 & 48.1 & 119 & 21.0 \\
\hline 2 or more & 11,872 & 52.7 & 263 & 79.2 & 19,495 & 51.9 & 447 & 79.0 \\
\hline Mean \pm S.D. & \multicolumn{2}{|c|}{$1.9+1.1$} & \multicolumn{2}{|c|}{$2.6+1.5$} & \multicolumn{2}{|c|}{$1.7+0.9$} & \multicolumn{2}{|c|}{$2.4+1.3$} \\
\hline Categorical & No. & $\%$ & No. & $\%$ & No. & $\%$ & No. & $\%$ \\
\hline Unmarried & 1,257 & 5.6 & 12 & 3.6 & 1,449 & 3.9 & 23 & 4.1 \\
\hline \multicolumn{9}{|l|}{ Employment status } \\
\hline Professional & 1,951 & 8.7 & 26 & 7.8 & 4,344 & 11.6 & 79 & 14.0 \\
\hline Managerial & 5,039 & 22.4 & 58 & 17.5 & 10,161 & 27.0 & 104 & 18.4 \\
\hline Skilled worker & 6,404 & 28.4 & 97 & 29.2 & 11,719 & 31.2 & 189 & 33.4 \\
\hline Semiskilled worker & 4,131 & 18.4 & 66 & 19.9 & 6,065 & 16.1 & 96 & 17.0 \\
\hline Unskilled worker & 1,735 & 7.7 & 41 & 12.3 & 2,141 & 5.7 & 46 & 8.1 \\
\hline Housewife & 2,368 & 10.5 & 38 & 11.4 & 2,315 & 6.2 & 39 & 6.9 \\
\hline Others & 883 & 3.9 & 6 & 1.8 & 840 & 2.2 & 13 & 2.3 \\
\hline \multicolumn{9}{|l|}{ Pregnancy supplements } \\
\hline Iron & 14,503 & 64.4 & 241 & 72.6 & 26,361 & 70.1 & 413 & 73.0 \\
\hline Calcium & 1,771 & 7.9 & 32 & 9.6 & 3,526 & 9.4 & 57 & 10.1 \\
\hline Folic acid & 11,806 & 49.2 & 193 & 58.1 & 20,449 & 54.4 & 326 & 57.6 \\
\hline Vitamin $B_{6}$ & 1,982 & 8.8 & 31 & 9.3 & 4,042 & 10.8 & 44 & 7.8 \\
\hline Vitamin D & 5,994 & 26.6 & 107 & 32.2 & 10,012 & 26.6 & 138 & 24.4 \\
\hline Vitamin C & 897 & 4.0 & 15 & 4.5 & 1,656 & 4.4 & 29 & 5.1 \\
\hline Vitamin E & 1,400 & 6.2 & 18 & 5.4 & 2,257 & 6.0 & 30 & 5.3 \\
\hline Multivitamin & 1,314 & 5.8 & 16 & 4.8 & 2,476 & 6.6 & 33 & 5.8 \\
\hline
\end{tabular}

\section{Statistical Analyses of Data}

We used SAS version 8.02 (SAS Institute Ins., Cary, North Carolina, USA) for statistical analyses. Contingency tables were prepared for the main study variables. First, the characteristics of pregnant women with VVLE were compared with all pregnant women without VVLE. X2 test was used for categorical variables, while Student t-test for quantitative variables. Second, pregnancy complications and maternal diseases, drug treatments were evaluated by ordinary logistic regression models and odds ratios (OR) with their 95\% confidence intervals (CI) were calculated. Third birth outcomes of control newborns without CA were compared in pregnant women with or without VVLE. Finally, we compared the prevalence of VVLE during the study pregnancy in specified CA groups with the prevalence of VVLE in their all matched control pairs. Adjusted OR with 95\% CI were evaluated in conditional logistic regression models for the occurrence of
VVLE. We examined confounding variables by comparing the OR for VVLE in the models with and without inclusion of the potential confounding variables. Finally, maternal age $(<20 \mathrm{yr}$, 20-29 yr, and $30 \mathrm{yr}$ or more), birth order (first delivery or one or more previous deliveries), maternal employment status and VVLE related drug treatments were included in the models as potential confounders.

\section{RESULTS}

The case group consisted of 22,843 malformed newborns or fetuses (“informative offspring”), and in 332 women (1.45\%) was VVLE medically recorded in the prenatal maternity logbooks. The total number of births in Hungary was 2,146,574 during the study period between 1980 and 1996, and 38,151 controls thus 
Table 2. Prevalence of medically recorded pregnancy complications in the study groups

\begin{tabular}{|c|c|c|c|c|c|c|c|c|}
\hline \multirow{5}{*}{ Pregnancy complications } & \multicolumn{4}{|c|}{ Case mothers } & \multicolumn{4}{|c|}{ Control mothers } \\
\hline & \multicolumn{2}{|c|}{ without } & \multicolumn{2}{|c|}{ with } & \multicolumn{2}{|c|}{ without } & \multicolumn{2}{|c|}{ with } \\
\hline & \multicolumn{4}{|c|}{ VVLE } & \multicolumn{4}{|c|}{ VVLE } \\
\hline & \multicolumn{2}{|c|}{$(\mathrm{N}=22,511)$} & \multicolumn{2}{|c|}{$(\mathrm{N}=332)$} & \multicolumn{2}{|c|}{$(\mathrm{N}=37,585)$} & \multicolumn{2}{|c|}{$(\mathrm{N}=566)$} \\
\hline & No. & $\%$ & No. & $\%$ & No. & $\%$ & No. & $\%$ \\
\hline Threatened abortion & 3,445 & 15.3 & 56 & 16.9 & 6,417 & 17.1 & 95 & 16.8 \\
\hline Nausea, vomiting, severe & 1,716 & 7.6 & 30 & 9.0 & 3.820 & 10.2 & 49 & 8.7 \\
\hline Preeclampsia-eclampsia & 1,727 & 7.7 & 41 & 12.3 & 3,103 & 8.3 & 56 & 9.9 \\
\hline Placental disorders & 294 & 1.3 & 3 & 0.9 & 582 & 1.5 & 11 & 1.9 \\
\hline Poly/oligohydramnios & 236 & 1.0 & 8 & 2.4 & 205 & 0.5 & 0 & 0.0 \\
\hline Threatened preterm delivery & 2,575 & 11.4 & 28 & 8.4 & 5,374 & 14.3 & 72 & 12.7 \\
\hline Gestational diabetes & 163 & 0.7 & 3 & 0.9 & 287 & 0.8 & 5 & 0.9 \\
\hline Anaemia & 3,166 & 14.1 & 74 & 22.3 & 6,235 & 16.6 & 121 & 21.4 \\
\hline
\end{tabular}

Table 3. The occurrence of VVLE related drug treatments in the study groups

\begin{tabular}{|c|c|c|c|c|c|c|c|c|}
\hline \multirow{5}{*}{ Drugs } & \multicolumn{4}{|c|}{ Case mothers } & \multicolumn{4}{|c|}{ Control mothers } \\
\hline & \multicolumn{2}{|c|}{ without } & \multicolumn{2}{|c|}{ with } & \multicolumn{2}{|c|}{ without } & \multicolumn{2}{|c|}{ with } \\
\hline & \multicolumn{4}{|c|}{ VVLE } & \multicolumn{4}{|c|}{ VVLE } \\
\hline & \multicolumn{2}{|c|}{$(\mathrm{N}=22,511)$} & \multicolumn{2}{|c|}{$(\mathrm{N}=332)$} & \multicolumn{2}{|c|}{$(\mathrm{N}=37,585)$} & \multicolumn{2}{|c|}{$(\mathrm{N}=566)$} \\
\hline & No. & $\%$ & No. & $\%$ & No. & $\%$ & No. & $\%$ \\
\hline Hydroxyaethylrutoside & 385 & 1.7 & 182 & 54.8 & 849 & 2.3 & 294 & 51.9 \\
\hline Phenylbutazone & 76 & 0.3 & 26 & 7.8 & 149 & 0.4 & 35 & 6.2 \\
\hline Rutoside & 140 & 0.6 & 90 & 27.1 & 405 & 1.1 & 144 & 25.4 \\
\hline Tribenoside & 139 & 0.6 & 35 & 10.5 & 217 & 0.6 & 68 & 12.0 \\
\hline
\end{tabular}

represented $1.8 \%$ of all Hungarian births. Of these 38,151 controls, 566 (1.48\%) had medically recorded VVLE.

Of 332 case mothers, 91 (27.4\%) had the onset of VVLE before conception, this condition was considered as chronic. Of 566 control mothers, 127 (22.4\%) were recorded with chronic VVLE. Most of these women $(96.7 \%$ of cases and $96.1 \%$ of control mothers) had previous pregnancies. The new onset VVLE was recorded after the third month with a peak in the fifth and sixth gestational month.

The main variables of mothers with VVLE compared with pregnant women without VVLE as reference are shown in Table 1. This comparison indicates a higher mean maternal age due to the larger proportion of women older than 30 years in older pregnant women with VVLE. The mean birth order was also much higher with the predominance with birth order 2 or more. Marital status did not show difference among the study groups. However, the proportion of managerial women was smaller while the proportion of unskilled workers (with mainly with standing working position) was larger in pregnant women with VVLE.

Among pregnancy supplements, here only folic acid and folic acid-containing multivitamins are mentioned. Folic acid supplementation during pregnancy was more frequent in pregnant women with VVLE. The frequency of multivitamin users was similar in both study groups.
In the study group, 2,640 pregnant women were visited at home and their smoking habit was evaluated on the basis of "family consensus". VVLE were recorded in 40 (1.5\%) pregnant women, and $6(15.0 \%)$ smoked. Of 2,600 pregnant women without VVLE, 567 (21.8\%) smoked. Of 800 control mothers visited at home, 11 (1.4\%) had recorded VVLE, and 2 smoked during the study pregnancy. The rest, i.e. of 789 pregnant women, 152 (19.0\%) were smokers.

Among acute maternal diseases, only the incidence of influenza-common cold (in general with secondary complications) showed some difference among the study groups, it was less frequent in both case (14.8\% vs. $21.8 \%)$ and control (15.9\% vs. $18.5 \%)$ mothers with VVLE than in pregnant women without VVLE. The well-known important chronic diseases such as diabetes mellitus, epilepsy, etc, did not show difference in their prevalence among the study groups. However, the prevalence of constipation was $5.4 \%$ in case mothers and 3.9\% in control mothers with VVLE compared to the $2.0 \%$ of pregnant women without VVLE. The prevalence of haemorrhoid was much higher in case mothers (15.4\%) and control mothers (20.8\%) with VVLE than in pregnant women without VVLE (3.7\%).

At the evaluation of pregnancy complications only medically recorded conditions were considered (Table 2). The incidence of preeclampsia-eclampsia was somewhat higher while the rate of 
Table 4. Birth outcomes of control newborn infants without CA born to mothers with or without VVLE

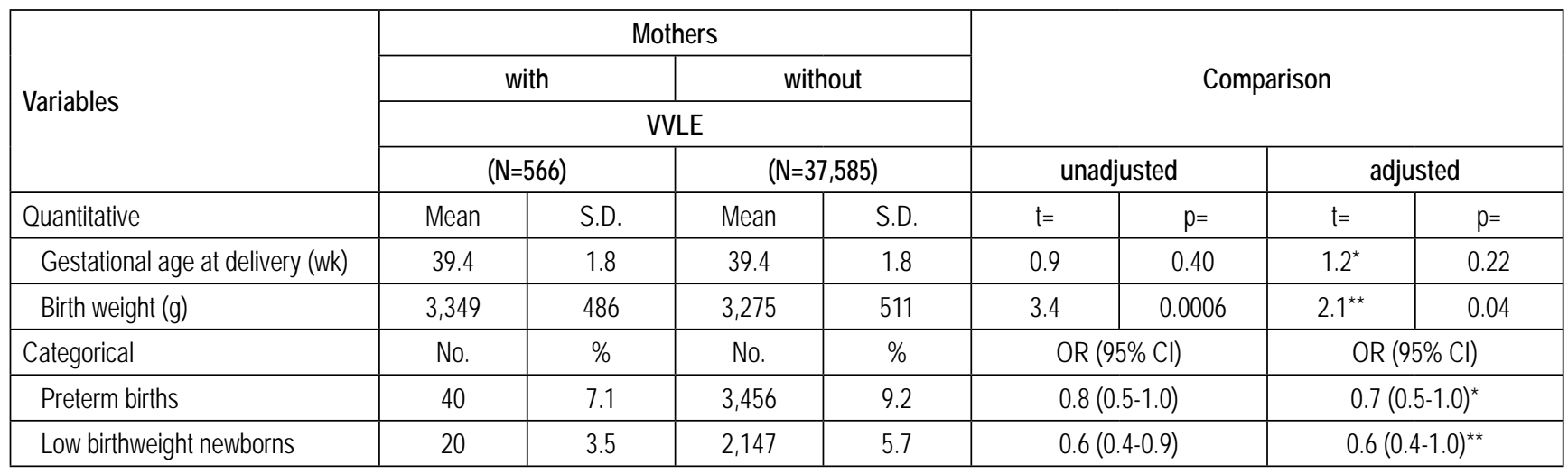

*adjusted for maternal age, birth order and maternal socio-economic status

**adjusted for maternal age, birth order, maternal socio-economic status and gestational age

Table 5. Results of risk estimation in cases with different CAs and their all matched controls born to mothers with VVLE

\begin{tabular}{|c|c|c|c|c|c|c|c|c|c|}
\hline \multirow{2}{*}{ Study groups } & \multirow{2}{*}{$\begin{array}{c}\text { Grand total } \\
\text { No. }\end{array}$} & \multicolumn{4}{|c|}{ Any time during pregnancy } & \multicolumn{4}{|c|}{ First trimester } \\
\hline & & \multirow{2}{*}{$\begin{array}{l}\text { No } \\
566\end{array}$} & \multirow{2}{*}{$\begin{array}{c}\% \\
1.48\end{array}$} & OR* & $95 \% \mathrm{Cl}$ & \multirow{2}{*}{$\begin{array}{l}\text { No } \\
210\end{array}$} & \multirow{2}{*}{$\begin{array}{c}\% \\
0.6\end{array}$} & OR* & $95 \% \mathrm{Cl}$ \\
\hline Controls & 38,151 & & & \multicolumn{2}{|c|}{ reference } & & & \multicolumn{2}{|c|}{ reference } \\
\hline Isolated CAs & & & & & & & & & \\
\hline Neural-tube defects & 1,202 & 13 & 1.08 & 1.1 & $0.5-2.2$ & 4 & 0.3 & 0.5 & $0.2-1.7$ \\
\hline Cleft lip +/- palate & 1,375 & 18 & 1.31 & 0.6 & $0.3-1.1$ & 4 & 0.3 & 0.3 & $0.1-0.8$ \\
\hline Cleft palate & 601 & 9 & 1.50 & 1.4 & $0.5-3.5$ & 3 & 0.5 & 1.8 & $0.3-9.2$ \\
\hline Oesophageal atresia/stenosis & 217 & 3 & 1.38 & 0.6 & $0.1-2.3$ & 3 & 1.4 & 1.5 & $0.3-7.9$ \\
\hline Rectal/anal atresia/stenosis & 231 & 2 & 0.87 & 0.6 & $0.1-3.8$ & 0 & 0.0 & - & - \\
\hline Obstructive CAs of urinary tract & 343 & 3 & 0.87 & 1.4 & $0.3-7.5$ & 2 & 0.6 & 1.5 & $0.2-11.9$ \\
\hline Hypospadias & 3,038 & 36 & 1.18 & 0.8 & $0.6-1.2$ & 13 & 0.4 & 0.7 & $0.3-1.3$ \\
\hline Undescended testis & 2,052 & 31 & 1.51 & 1.1 & $0.7-1.8$ & 12 & 0.6 & 1.3 & $0.6-3.0$ \\
\hline Exomphalos/gastroschisis & 255 & 2 & 0.78 & 0.9 & $0.2-4.7$ & 1 & 0.4 & 2.1 & $0.1-40.1$ \\
\hline Microcephaly, primary & 111 & 2 & 1.80 & 0.3 & $0.1-1.8$ & 0 & 0.0 & - & - \\
\hline Hydrocephaly, congenital & 314 & 2 & 0.64 & 0.6 & $0.1-2.9$ & 2 & 0.6 & 3.3 & $0.3-40.8$ \\
\hline Eye CAs & 99 & 3 & 3.03 & 1.6 & $0.9-4.4$ & 2 & 2.0 & 3.3 & $0.2-44.4$ \\
\hline EarCAs & 354 & 9 & 2.54 & 2.1 & $0.7-5.9$ & 3 & 0.9 & $1.5-$ & $0.6-2.5$ \\
\hline Cardiovascular CAs & 4,480 & 71 & 1.58 & 0.9 & $0.7-1.3$ & 32 & 0.7 & 1.2 & $0.7-2.0$ \\
\hline Clubfoot & 2,424 & 44 & 1.82 & 1.2 & $0.8-1.8$ & 18 & 0.7 & 1.4 & $0.7-2.6$ \\
\hline Limb deficiencies & 548 & 5 & 0.91 & 0.7 & $0.2-2.0$ & 1 & 0.2 & 0.3 & $0.0-2.9$ \\
\hline Poly/syndactyly & 1,744 & 30 & 1.72 & 1.1 & $0.7-1.7$ & 12 & 0.7 & 1.1 & $0.5-2.4$ \\
\hline CAs of musculo-skeletal system & 585 & 10 & 1.71 & 1.2 & $0.4-2.1$ & 5 & 1.0 & 1.4 & $0.4-4.7$ \\
\hline Diaphragmatic CAs & 244 & 4 & 1.64 & 2.2 & $0.6-8.8$ & 1 & 0.4 & 0.5 & $0.1-5.2$ \\
\hline Other isolated CAs & 1,277 & 9 & 0.70 & 0.5 & $0.2-1.1$ & 2 & 0.2 & 0.2 & $0.0-1.0$ \\
\hline Multiple CAs & 1,349 & 26 & 1.93 & 0.9 & $0.5-1.4$ & 15 & 1.1 & 1.2 & $0.6-2.5$ \\
\hline Total & 22,843 & 332 & 1.45 & 0.9 & $0.8-1.1$ & 135 & 0.6 & 1.0 & $0.8-1.2$ \\
\hline
\end{tabular}

*OR adjusted for maternal age and employment status, birth order and related drug treatments in conditional logistic regression models

anemia was significantly higher in pregnant women with VVLE than in pregnant women without VVLE. On the other hand the rate of threatened preterm delivery was lower in pregnant women with VVLE compared to pregnant women without VVLE.

Among drugs, only VVLE related treatments such as hydroxyaethylrutoside (Venoruton ${ }^{\circledR}$ both for oral and local treatment as gel), rutoside (Rutascorbin ${ }^{\circledR}$ : rutoside and ascorbic acid), tribenoside (for oral treatment) and phenylbutazone (ointment for local treatment) showed obvious difference between case and control mothers with or without VVLE (Table 3). About two-third of case and control pregnant women were treated by these drugs, but there was no significant difference in the frequency of their uses 
Table 6. Data of cases with pectus excavatum

\begin{tabular}{|c|c|c|c|c|c|c|}
\hline Sex & Gestational week & $\begin{array}{l}\text { Birth weight } \\
\text { (g) }\end{array}$ & $\begin{array}{c}\text { VVLE } \\
\text { (months) }\end{array}$ & $\begin{array}{l}\text { Treatment } \\
\text { (months) }\end{array}$ & $\begin{array}{l}\text { Other diseases } \\
\text { (months) }\end{array}$ & $\begin{array}{l}\text { Other treatments } \\
\text { (months) }\end{array}$ \\
\hline M & 39 & 3,400 & VII-IX & Venoruton ${ }^{\circledast}(\mathrm{V} \mid \mathrm{-}-\mathrm{IX})$ & $\begin{array}{c}\text { Threatened } \\
\text { abortion/preterm } \\
\text { delivery (II-VII) }\end{array}$ & $\begin{array}{l}\text { Promethazine } \\
\quad(I I-V I I)\end{array}$ \\
\hline$M$ & 36 & 2,800 & V-IX & Venoruton ${ }^{\circledR}(\mathrm{V}-\mathrm{IX})$ & - & - \\
\hline $\mathrm{F}$ & 41 & 3,100 & V-IX & - & - & - \\
\hline $\mathrm{F}$ & 38 & 3,000 & I-IX & - & - & - \\
\hline$M$ & 39 & 2,700 & I-IX & Venoruton $^{\circledast}(\mathrm{I}-\mathrm{IX})$ & - & - \\
\hline M & 39 & 3,850 & $|-| X$ & Venoruton $^{\circledast}(\mathrm{V}-\mathrm{IX})$ & $\begin{array}{l}\text { Acute infection of } \\
\text { urinary tract (VI) }\end{array}$ & Cotrimoxazole (VI) \\
\hline
\end{tabular}

$M=$ male; $F=$ female; Venoruton ${ }^{\circledR}=$ hydroxyaethylrutoside

between case and control mothers. Surgical intervention due to VVLE occurred only in two women during the study pregnancy.

The birth outcomes of control newborns are shown in Table 4, the data of cases are omitted because CAs have a more drastic effect for these variables than maternal VVLE. There was no difference in the sex ratio between the study groups. The mean gestational age at delivery did not show any difference between newborn infants born to mothers with or without VVLE. However, the newborn infants born to pregnant women with VVLE were as average, $74 \mathrm{~g}$ heavier. In addition the rate of both preterm births and of low birthweight newborns was lower in the group of pregnant women with VVLE compared to the children of mothers without VVLE. However, mean birth weight, the rate of preterm birth and low birthweight were not statistically different in the newborns of pregnant women with VVLE compared to newborns of pregnant women without VVLE on the basis of adjusted OR.

The major objective of the study was the evaluation of the possible association of pregnant women with VVLE and the risk of different CAs in their recorded offspring. At the estimation of possible risk for CAs, first the occurrence of VVLE any time during pregnancy was compared between cases with 21 different CA-groups including at least 2 cases and their all matched controls (Table 5). The total rate of cases with CA and any specified CA-group did not show any risk.

In the next step the prevalence of VVLE only in the critical period of major CA, i.e. in the second and/or third gestational month and during the first trimester was evaluated (because if pregnant women had chronic VVLE, i.e. before conception of the study pregnancy, it continued at the end of pregnancy). The total rate of cases with CA and any specified CA-group did not show any risk.

Finally CAs and subgroups of CAs within CA-groups were analyzed separately. The group of CA of musculo-skeletal system included 10 cases born to mothers with VVLE and among them 6 cases were affected with pectus excavatum. The total group of CAs of musculo-skeletal system comprised 585 cases, within this group 82 had pectus excavatum. Thus the percentage figure of pectus excavatum was $0.36 \%$ among 22,843 cases with CAs. Of 332 cases with CA born to mother with VVLE, 6 (1.81\%) were affected with pectus excavatum, i.e. there is 5 fold higher risk for this CA in the children of mothers with VVLE $\left(\chi_{1}^{2}=19.3\right.$, $\mathrm{p}<0.0001$ ) This association was confirmed by the comparison of 6 cases with pectus excavatum and their matched controls according the prevalence of VVLE in their mothers (OR with 95\% CI: 5.4, 2.3-12.4). The detailed analysis of 4 males and 2 females with pectus excavatum did not reveal any potential teratogenic effect, 4 were treated with Venoruton ${ }^{\circledR}$ (Table 6).

\section{DISCUSSION}

The possible association between maternal VVLE and pregnancy complications and birth outcomes was evaluated in the study. Three findings are worth reporting. (i) Mothers with VVLE were older with higher parity and were more frequently unskilled workers than women without VVLE. This is in agreement with the findings in previous studies $(15,16)$. (ii) There was a higher risk for anaemia, constipation and haemorrhoids in pregnant women with VVLE compared with women without VVLE. (iii) The risk of pectus excavatum was 5- fold higher in the children of pregnant women with VVLE compared with the children of women without VVLE.

The prevalence of VVLE was 1.2-2.0\% of Hungarian pregnant women and this figure is much lower than about $40 \%$ of VVLE rate reported in previous studies (15-17). However, the prevalence of VVLE in pregnant women depends on the age and parity of females, in addition to the diagnostic criteria of VVLE. In our study only severe and in general pregnant women treated with VVLE were recorded in the prenatal maternity logbook, thus obviously this group of mothers with VVLE may not reflect all women with VVLE.

Only a quarter of VVLE occurred before the study pregnancy, most in multiparae, while in general new onset VVLE manifested in the second trimester of pregnancy. However, it is difficult to differentiate the real onset of VVLE and the onset of complaints in these pregnant women. The higher risk of VVLE in pregnant women may be connected with the hormonal changes for arterio-venosus shunt, the mechanical effect of growing uterus for abdominal veins, maternal weight gain with some fluid retention (15). Thus pregnancy is an important causal factor in the manifestation of VVLE, and it partly explains that VVLE at least is twice as frequent in females as in males (1).

Our pregnant women with VVLE had a higher occurrence of anaemia partly related to haemorrhoids and constipation which were 4.2-5.6 times higher in case and control pregnant women 
with VVLE than in pregnant women without VVLE and the bleeding in pregnant women with haemorrhoids was frequently recorded. The diagnosis of anemia was based on the number of erythrocytes, hemoglobin and haematocrit because these laboratory tests were performed in the prenatal care clinics. In Hungary most anaemias during pregnancy are caused by iron deficiency, and 73\% of pregnant women with VVLE were treated with iron products.

There was no higher risk for adverse birth outcomes in pregnant women with VVLE compared with women without VVLE. In fact the rate of preterm births and low birthweight was somewhat lower in the newborns of pregnant women with VVLE and it may be connected with better prenatal care and the higher use of folic acid.

Among CAs, only pectus excavatum associated with a higher risk in children of pregnant women with VVLE compared with the children of women without VVLE. Previous epidemiological studies were frequently based on the evaluation of total CAs which is a very insensitive variable including about 200 CAs with heterogeneous origins. Recently CA-groups with similar anatomic manifestation and with more-or-less similar etiology have been evaluated, e.g. in 25 CA-groups in the HCCSCA. However, as this study shows the grouping of similar manifestation of musculo-skeletal CAs is also too heterogeneous and may cover up sub-entities with significant association with etiological factors. Thus special computer programs are needed in human teratology which can reveal significant associations in specific entities as well within the evaluated CA-groups (18).

Pectus excavatum (funnel chest) is a depression deformity of the thorax in which the sternum, usually the lower part, is depressed towards the spine. This condition may be evident at birth, but in the majority of cases it first becomes noticeable when the infant has several months of age (19). The severity spectrum of pectus excavatum is wide starting from the mild manifestation which presents only cosmetic problem, to the severe deformation with impaired ventilator capacity of lungs and displacement of the heart, which in general requires surgical intervention. Our cases were selected from HCAR to the HCCSCA within the first three postnatal months, thus most cases might had severe manifestation of pectus excavatum.

A certain part of pectus excavatum has autosomal dominant inheritance, but most cases are sporadic. Hauge and Gundersen (20) presented a family study of 249 probands with VVLE, and they concluded that multifactorial inheritance seems very probable. Later Matousek and Prerovsky (21) estimated the heritability of varicose veins as about $50 \%$ within multifactorial origin. The question is whether the possible association of maternal VVLE with the higher risk for pectus excavatum is causal or can be explained by related drug treatments, unevaluated confounders or by chance.

The biological plausibility that maternal VVLE can cause pectus excavatum in the offspring is minimal. However, both VVLE and pectus excavatum are connected with the tissue of mesodermal origin thus may have common genetic origin. According to the aphorism of Osler: "Varicose veins are the results of an improper selection of grandparents" (22). Thus, it is possible that the association of VVLE and pectus excavatum is connected with the improper selection of parents.

The recommended first line therapy of VVLE includes dietary changes, losing weight, smoking cessation and the education for the proper position of lower limb in general, but particularly during relaxation. The second line of therapy comprises of compressive bandage and some drugs. Drugs used for the treatment of VVLE do not seem to be teratogenic though the teratogenic effect of hydroxyaethylrutoside was shown in the origin of ocular coloboma (23). As far as we know the teratogenic potential of tribenisode has not been studied (3). The teratogenic effect of phenylbutazone was not found in animal investigations (24, 25), but Kullander and Kallen (26) reported that 18 women taking phenylbutazone in the first trimester had one miscarriage, $6 \mathrm{mi}$ nor and one major CA. The third line of therapy is sclerotherapy and surgical intervention (ablation of the veins) but it was not recommended in Hungarian pregnant women. The point is that the association of VVLE related drugs treatment with a higher risk of pectus excavatum is unlikely.

Another possible explanation for this association is unevaluated/unknown confounders beyond the evaluated confounders. Finally multiple comparisons may produce non-causal association because significant difference is expected in every 20th estimation due to chance. This possible association was based only on 6 cases and we evaluated 21 CA-groups thus after the Bonferroni adjustment (27) the significant p-value was reduced to 0.002 . Thus, this association has remained significant, nevertheless needs confirmation or rejection in other studies.

The strengths of the HCCSCA are that is a population-based large data set including 898 pregnant women with prospectively and medically recorded severe VVLE in an ethnically homogeneous Hungarian (Caucasian) population. Additional strengths are matching of cases to controls without CA; available data for potential confounders, medically recorded gestational age at delivery, birth weight and pregnancy complications, and finally that the diagnosis of medically reported CAs was checked in the HCAR (5) and later modified, if necessary, on the basis of recent medical examination within the HCCSCA (4).

However, this data set also has limitations. (i) We did not know any details regarding the onset of chronic VVLE before pregnancy. (ii) Other pregnancy outcomes, e.g. miscarriages were not known. (iii) The lifestyle of pregnant women was also not known in the total data set due to the unreliability of maternal self-reported data regarding smoking and drinking during the studied pregnancy (28), thus these data were obtained in a subsample based on cross interview of mothers and fathers at the home visit. However, smoking and drinking habit had no role in the origin of pectus excavatum, and our data showed a lower rate of smokers among pregnant women with VVLE.

In conclusion, a higher risk for pectus excavatum was found in the children of pregnant women with VVLE though other adverse birth outcomes have not been revealed, thus VVLE in pregnant women does not associate with other obvious hazard for their fetuses.

\section{REFERENCES}

1. Wennberg PW, Rooke TW. Diagnosis and management of diseases of the peripheral arteries and veins. In: Fuster V, Walsh RA, O’Rourke RA, Poole-Wilson P, editors. Hurst's the heart. 12th ed. New York: McGraw Hill Medical; 2008. p. 2371-88.

2. Wong RC, Ellis CN. Physiologic skin changes in pregnancy. Semin Dermatol. 1989 Mar;8(1):7-11.

3. Shepard TH, Lemire RJ. Catalog of teratogenic agents. 11th ed. Baltimore: Johns Hopkins University Press; 2004. 
4. Czeizel AE, Rockenbauer M, Siffel C, Varga E. Description and mission evaluation of the Hungarian case control surveillance of congenital abnormalities, 1980-1996. Teratology. 2001 May;63(5):176-85.

5. Czeizel AE. The first 25 years of the Hungarian congenital abnormality registry. Teratology. 1997 May;55(5):299-305.

6. Czeizel AE, Intôdy Z, Modell B. What proportion of congenital abnormalities can be prevented? BMJ. 1993 Feb 20;306(6876):499-503. Erratum in: BMJ 1993 Mar 20;306(6880):771.

7. Czeizel AE, Petik D, Vargha P. Validation studies of drug exposures in pregnant women. Pharmacoepidemiol Drug Saf. 2003 JulAug;12(5):409-16.

8. Czeizel AE, Vargha P. Periconceptional folic acid/multivitamin supplementation and twin pregnancy. Am J Obstet Gynecol. 2004 Sep;191(3):790-4.

9. Beebe HG, Bergan JJ, Bergqvist D, Eklof B, Eriksson I, Goldman MP, et al. Classification and grading of chronic venous disease in the lower limbs. A consensus statement. Eur J Vasc Endovasc Surg. 1996 Nov;12(4):48790; discussion 491-2.

10. Eklöf B, Rutherford RB, Bergan JJ, Carpentier PH, Gloviczki P, Kistner RL, et al; American Venous Forum International Ad Hoc Committee for Revision of the CEAP Classification. Revision of the CEAP classification for chronic venous disorders: consensus statement. J Vasc Surg. 2004 Dec;40(6):1248-52.

11. Marik PE, Plante LA. Venous thromboembolic disease and pregnancy. N Engl J Med. 2008 Nov 6;359(19):2025-33.

12. Puhó E, Métneki J, Czeizel AE. Maternal employment status and isolated orofacial clefts in Hungary. Cent Eur J Public Health. 2005 Sep;13(3):144-8.

13. Czeizel AE, Puhó EH, Ács N, Bánhidy F. Use of specified critical periods of different congenital abnormalities instead of the first trimester concept. Birth Defects Res A Clin Mol Teratol. 2008 Mar;82(3):139-46.

14. Czeizel AE. Specified critical period of different congenital abnormalities: a new approach for human teratological studies. Congenit Anom (Kyoto). 2008 Sep;48(3):103-9.

15. Kroeger K, Ose C, Rudofsky G, Roesener J, Hirche H. Risk factors for varicose veins. Int Angiol. 2004 Mar;23(1):29-34.

16. Kurz X, Lamping DL, Kahn SR, Baccaglini U, Zuccarelli F, Spreafico $\mathrm{G}$, et al; VEINES Study Group. Do varicose veins affect quality of life?
Results of an international population-based study. J Vasc Surg. 2001 Oct;34(4):641-8.

17. Evans CJ, Fowkes FG, Ruckley CV, Lee AJ. Prevalence of varicose veins and chronic venous insufficiency in men and women in the general population: Edinburgh Vein Study. J Epidemiol Community Health. 1999 Mar;53(3):149-53.

18. Czeizel AE. The estimation of human teratogenic/fetotoxic risk of exposures to drugs on the basis of Hungarian experience: a critical evaluation of clinical and epidemiological models of human teratology. Expert Opin Drug Saf. 2009 May;8(3):283-303.

19. Verhagen AD. Funnel chest. In: Rubin A, editor. Handbook of congenital malformations. Philadelphia: W.B. Saunders Company; 1969. p. 157-8.

20. Hauge M, Gundersen J. Genetics of varicose veins of the lower extremities. Hum Hered. 1969;19(5):573-80.

21. Matousek V, Prerovský I. A contribution to the problem of the inheritance of primary varicose veins. Hum Hered. 1974;24(3):225-35.

22. Bean RB, Bean WB, editors. Sir William Osler. Aphorisms from his bedside teachings and writings. New York: Henry Schuman Publ; 1950.

23. Vogt G, Puhó E, Czeizel AE. A population-based case-control study of isolated ocular coloboma. Ophthalmic Epidemiol. 2005 Jun;12(3):191-7.

24. Larsen V, Bredahl E. The embryotoxic effect on rabbits of monophenylbutazone (monazan) compared with phenylbutazone and thalidomide. Acta Pharmacol Toxicol (Copenh). 1966;24(4):443-5.

25. Schardein JL, Blatz AT, Woosley ET, Kaump DH. Reproduction studies on sodium meclofenamate i comparison to aspirin and phenylbutazone. Toxicol Appl Pharmacol. 1969 Jul;15:46-55.

26. Kullander S, Källén B. A prospective study of drugs and pregnancy. 4. Miscellaneous drugs. Acta Obstet Gynecol Scand. 1976;55(4):287-95.

27. Perneger TV. What's wrong with Bonferroni adjustments. BMJ. 1998 Apr 18;316(7139):1236-8.

28. Czeizel AE, Petik D, Puhó E. Smoking and alcohol drinking during pregnancy. The reliability of retrospective maternal self-reported information. Centr Eur J Public Health. 2004 Dec;12(4):179-83.

Received 9. 11. 2009 Accepted in revised form 10. 3. 2010 